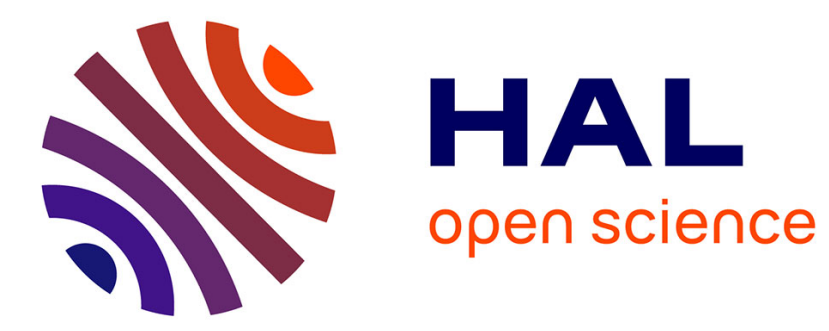

\title{
Dirac concentrations in a chemostat model of adaptive evolution
}

\author{
Alexander Lorz, Benoît Perthame, Cécile Taing
}

\section{To cite this version:}

Alexander Lorz, Benoît Perthame, Cécile Taing. Dirac concentrations in a chemostat model of adaptive evolution. Chinese Annals of Mathematics - Series B, 2017. hal-01255449

\section{HAL Id: hal-01255449 \\ https://hal.sorbonne-universite.fr/hal-01255449}

Submitted on 13 Jan 2016

HAL is a multi-disciplinary open access archive for the deposit and dissemination of scientific research documents, whether they are published or not. The documents may come from teaching and research institutions in France or abroad, or from public or private research centers.
L'archive ouverte pluridisciplinaire HAL, est destinée au dépôt et à la diffusion de documents scientifiques de niveau recherche, publiés ou non, émanant des établissements d'enseignement et de recherche français ou étrangers, des laboratoires publics ou privés. 


\title{
Dirac concentrations in a chemostat model of adaptive evolution
}

\author{
Alexander Lorz ${ }^{* \dagger} \quad$ Benoît Perthame ${ }^{* \dagger} \quad$ Cécile Taing ${ }^{* \dagger}$
}

October 27, 2015

\begin{abstract}
We consider parabolic systems of Lotka-Volterra type that describe the evolution of phenotypically structured populations. Nonlinearities appear in these systems to model interactions and competition phenomena leading to selection. In this paper, the equation on the structured population is coupled with a differential equation on the nutrient concentration that changes as the total population varies.

We review different methods aimed at showing the convergence of the solutions to a moving Dirac mass. Setting first two frameworks based on weak or strong regularity assumptions in which we study the concentration of the solution, we state $B V$ estimates in time on appropriate quantities and derive a constrained Hamilton-Jacobi equation to identify the Dirac locations.
\end{abstract}

Key-words: Adaptive evolution; Asymptotic behaviour; Chemostat; Dirac concentrations; Hamilton-Jacobi equations; Lotka-Volterra equations; Viscosity solutions.

AMS Class. No: 35B25, 35K57, 47G20, 49L25, 92D15.

\section{Introduction}

We survey several methods developed to study concentration effects in parabolic equations of Lotka-Volterra type. Furthermore, we extend the theory to a coupled system motivated by models of chemostat where we observe very rare mutations for a long time. These equations have been established with the aim of describing how speciation occurs in biological populations, taking into account competition for resources and mutations in the populations. There is a large literature on the subject where the mutation-competition principles are illustrated in various mathematical terms: for instance in [23, 28, 35] for an approach based on the study of the stability of differential systems, in [30, 29, 45] for the

\footnotetext{
*Sorbonne Universits, UPMC Univ Paris 06, Inria, Laboratoire Jacques-Louis Lions UMR CNRS 7598, F75005 Paris, France

${ }^{\dagger}$ Emails: lorz@ann.jussieu.fr, benoit.perthame@upmc.fr, taing@ann.jussieu.fr
} 
evolutionary games theory, in [14] for the study of stochastic individual based models, or in 6, 36, 42 for the study of integro-differential models. We choose here the formalism using parabolic partial differential equations, widely developed in [5, 7, 21, 41] to describe the competition dynamics in a chemostat.

The chemostat is a bioreactor to which fresh medium containing nutrients is continuously added, while culture liquid is continuously removed to keep the culture volume constant. This device is used as an experimental ecosystem in evolutionary biology to observe mutation and selection processes driven by competition for resources. From the mathematical point of view, the theoretical description of the population dynamics in a chemostat leads to highly nonlinear models and questions of long term behaviour and convergence to an evolutionary steady state naturally arise (see [1, 19, 24, 39, 44]).

Our aim is to study a generalization of the chemostat model introduced in [34] with a representation of mutations by a diffusion term. In this model, each individual in the population is characterized by a quantitative phenotypic trait $x \in \mathbb{R}^{d}$ and $n_{\varepsilon}(t, x)$ denotes the population density at time $t$ with the trait $x$. We study the following equations

$$
\begin{aligned}
& \varepsilon \partial_{t} n_{\varepsilon}(x, t)=n_{\varepsilon} R\left(x, S_{\varepsilon}(t)\right)+\varepsilon^{2} \Delta n_{\varepsilon}(x, t), \quad x \in \mathbb{R}^{d}, t \geq 0 \\
& \varepsilon \beta \frac{d}{d t} S_{\varepsilon}(t)=Q\left(S_{\varepsilon}(t), \rho_{\varepsilon}(t)\right), \\
& \rho_{\varepsilon}(t):=\int_{\mathbb{R}^{d}} n_{\varepsilon}(x, t) d x
\end{aligned}
$$

where the function $R\left(x, S_{\varepsilon}\right)$ represents a trait-dependent birth-death rate and $S_{\varepsilon}$ denotes the nutrient concentration which changes over time with rate $Q$. Here $\varepsilon$ is a small parameter which allows to consider very rare mutations and large times of order $\varepsilon^{-1}$. The idea of a $\varepsilon^{-1}$ rescaling in the space and time variables goes back to [31, 32, to study propagation for systems of reaction-diffusion PDE. The parameter $\beta$, introduced first in [34, gives a time scale which, as $\beta \rightarrow 0$, leads to the equation $Q(\rho, S)=0$ and in this case, under suitable assumptions, we deduce the existence of a function $f$ by Implicit Function Theorem such that $S=f(\rho)$ and the concentration results are known to hold [7, 33.

Such models can be derived from stochastic individual based models in the limit of large populations (refer to [16, 17]).

A possible way to express mathematically the emergence of the fittest traits among the population is to prove that $n_{\varepsilon}$ concentrates as a Dirac mass centred on some point $\bar{x}$ (or a sum of Dirac masses) when $\varepsilon$ vanishes, which means the phenotypic selection of a quantitative trait denoted by $\bar{x}$ in long times. The main results of the paper can be summarized as

Theorem 1.1. For well-prepared initial data and two classes of assumptions (monotonic in one dimension or concavity in multi-dimensions), then the concentration effect holds

$$
n_{\varepsilon}(t, x) \underset{\varepsilon \rightarrow 0}{\longrightarrow} \bar{\rho}(t) \delta(x-\bar{x}(t)) \quad \text { in the sense of measure }
$$


where the pair $(\bar{x}(t), \bar{\rho}(t))$ can be determined thanks to a constrained Hamilton-Jacobi equation given later on.

In order to describe these concentration effects and following earlier works on similar issues [7, 5, 13, 33, 18, 41], we will use the Hopf-Cole transformation defining $u_{\varepsilon}(t, x)=$ $\varepsilon \ln n_{\varepsilon}(t, x)$ and derive a Hamilton-Jacobi equation. Then we obtain by passing to the limit $\varepsilon \rightarrow 0$ a constrained Hamilton-Jacobi equation, whose solutions have a maximum value of 0 . The point is that the concentration locations in the limit $\varepsilon \rightarrow 0$ can be identified among the maximum points of these solutions. This method, introduced in [24] and used for instance in [42, 43] is very general and has been extended to various systems (see for the case of reaction-diffusion systems).

Singular perturbation problems in PDEs is a classical subject that has been studied from different viewpoints. For instance a seminal paper on parabolic equations involving measures is [11]. Also the above rescaling in parabolic equations or systems has been deeply studied in reaction-diffusion equations (see [4, 25]) leading to front propagation where a state invades another as in the Fishher-KPP equation where the stable state $n_{\varepsilon}=1$ invades the unstable state $n_{\varepsilon}=0$. This is also the case of Ginzburg-Landau equations (see [8]) where the quadratic observable $n_{\varepsilon}=\left|u_{\varepsilon}\right|^{2}$ takes asymptotically the value 1 . This is different from our case, as one can see in the above theorem and since we essentially derive $L^{1}$ bounds from the presented model.

To prove the main convergence results of this paper, we will adapt the method introduced in [7, 5, 34] to find $B V$ estimates for the appropriate quantities as a first step. Then we will use the theory of viscosity solutions to Hamilton-Jacobi equations (see [2, 3, 20, 27] for general introduction to this theory) to obtain the Dirac locations. In the first part we will proceed with assumptions of weak regularity of the growth rate in a first instance and then we will resume the study under concavity assumptions.

The paper is organized as follows. We first state (section 2) the framework of the general weak theory and its main results. We start the study by establishing $B V$ estimates on $\rho_{\varepsilon}^{2}$ and $S_{\varepsilon}$ in section 3. Section 4 is devoted to the analysis of the solutions to the constrained Hamilton-Jacobi equations. We first prove some regularity results for $u_{\varepsilon}$. Then we study the asymptotic behaviour of $u_{\varepsilon}$ and deduce properties of the concentration points. In section 5 we set the simple case of our results when the dimension $d$ equals 1 and prove concentration effects. In section 6 we review the $d$-dimensional framework where we assume uniform concavity of the growth-rate and initial conditions. We establish again the $B V$ estimates in this specific case and prove the uniform concavity of $u_{\varepsilon}$. The regularity obtained for $u_{\varepsilon}$ allow us to derive the dynamics of the concentration points in the form of a canonical equation. We complete these results by numerics in section 7 .

\section{The weak theory: assumptions and main results}

First of all, we give some assumptions to set a framework for the general weak theory. We use the same assumptions as [34. 
For the Lipschitz continuous functions $R$ and $Q$, we assume that there are constants $S_{0}>0, K_{Q}>0, \underline{K}_{1}>0$ and $\bar{K}_{1}>0$ such that

$$
\begin{aligned}
& Q(0, \rho)>0, \quad \max _{\rho \geq 0} Q\left(S_{0}, \rho\right)=0, \quad Q_{S}(S, \rho) \leq-K_{Q}, \quad Q_{\rho}(S, \rho) \leq-K_{Q}, \\
& 0<\underline{K}_{1} \leq R_{S}(x, S) \leq \bar{K}_{1}, \\
& \sup _{0 \leq S \leq S_{0}}\|R(\cdot, S)\|_{W^{2, \infty}\left(\mathbb{R}^{d}\right)} \leq K_{2} .
\end{aligned}
$$

We complete the system with the initial conditions $S^{0}, n_{\varepsilon}^{0}$ such that

$$
S_{m}<S^{0}<S_{0}, \quad n^{0}(x)>0, \forall x \in \mathbb{R}^{d}, \quad 0<\rho_{m} \leq \rho_{\varepsilon}^{0}:=\int_{\mathbb{R}^{d}} n_{\varepsilon}^{0}(x) d x \leq \rho_{M},
$$

where $\rho_{m}, \rho_{M}$ and $S_{m}$ are defined below.

We add to these assumptions a smallness condition on $\beta$ which can be written as

$$
\min _{\substack{0 \leq \rho \leq \rho_{M}, S_{m} \leq S \leq S_{0}}} \frac{\left|Q_{S}\right|}{\left|Q_{\rho}\right|} \geq 4 \beta \quad \max _{0 \leq \rho \leq \rho_{M},} \quad \frac{\bar{K}_{1} \rho_{M}}{\left|Q_{S}\right|},
$$

with the definition of $\rho_{M}$ stated below.

Note that from assumption (3), we directly obtain the bounds

$$
n_{\varepsilon}(t, x)>0, \quad 0<S_{\varepsilon}(t) \leq S_{0} .
$$

First we recall the following lemma, whose proof is given in [34]:

Lemma 2.1. Under the assumptions (3)-(6), there are constants $\rho_{m}, \rho_{M}$ and $S_{m}>0$ such that

$$
0<\rho_{m} \leq \rho_{\varepsilon}(t) \leq \rho_{M} \quad \text { and } \quad S_{m} \leq S_{\varepsilon}(t) \leq S_{0},
$$

where the value $S_{m}<S_{0}$ is defined by $Q\left(S_{m}, \rho_{M}\right)=0$.

This result is required to prove the following theorem.

Theorem 2.2. Assuming also (7), $\rho_{\varepsilon}(t)$ and $S_{\varepsilon}(t)$ have locally bounded total variation uniformly in $\varepsilon$. Consequently, there are limit functions $\rho_{m} \leq \bar{\rho} \leq \rho_{M}, S_{m} \leq \bar{S} \leq S_{0}$ such that, after extraction of a subsequence, we have

$$
S_{\varepsilon_{k}}(t) \underset{\varepsilon_{k} \rightarrow 0}{\longrightarrow} \bar{S}(t) \quad \text { and } \quad \rho_{\varepsilon_{k}}(t) \underset{\varepsilon_{k} \rightarrow 0}{\longrightarrow} \bar{\rho}(t), \quad \text { a.e. },
$$

and

$$
Q(\bar{\rho}, \bar{S})=0 \quad \text { a.e. }
$$


The next section is devoted to the proof of Theorem 2.2. Contrary to what we could expect, the establishment of the $B V$ estimates will be more complicated than in the previous works (see [7, 33]) where the nutrients are represented by an integral term as $\int \psi(x) n_{\varepsilon}(t, x) d x$. Here the main challenge comes from the equation (2) that we also have to consider to obtain $B V$ estimates on $S_{\varepsilon}$. An other difficulty comes from the parameter $\beta$. For $\beta$ large, it seems that we cannot derive $B V$ estimates with our approach and we expect oscillations of $S_{\varepsilon}$ and $\rho_{\varepsilon}$. This is the case for inhibitory integrate-and-fire models (see [12]) where delays generate periodic solutions. In the following proofs, $C$ denotes a constant which may change from line to line.

\section{$3 \quad$ BV estimates on $\rho_{\varepsilon}^{2}(t)$ and $S_{\varepsilon}(t)$}

\subsection{Bounds for $\rho_{\varepsilon}$}

We follow the lines of [34] to give the bounds $\rho_{m}$ and $\rho_{M}$. By integrating the equation (1) and using the assumptions (4) and (5), we arrive to the inequalities

$$
\varepsilon \frac{d}{d t} \rho_{\varepsilon} \leq \rho_{\varepsilon}\left(K_{2}+\overline{K_{1}} S_{\varepsilon}\right)
$$

and

$$
\varepsilon \frac{d}{d t} \ln \rho_{\varepsilon} \leq K_{2}+\overline{K_{1}} S_{0}
$$

Notice that $Q\left(S_{\varepsilon}, \rho_{\varepsilon}\right) \leq-K_{Q} \rho_{\varepsilon}+Q(0,0)$ from the assumptions in (3). By adding the equation (2) to the inequation above, we arrive to

$$
\begin{aligned}
\varepsilon \frac{d}{d t}\left(\ln \rho_{\varepsilon}+\beta S_{\varepsilon}\right) & \leq K_{2}+\overline{K_{1}} S_{0}+Q(0,0)-K_{Q} \rho_{\varepsilon} \\
& \leq K_{2}+\overline{K_{1}} S_{0}+Q(0,0)-\frac{K_{Q}}{e^{\beta S_{0}}} e^{\ln \rho_{\varepsilon}+\beta S_{\varepsilon}}
\end{aligned}
$$

It follows that, for $C_{2}$ the root in $\ln \rho_{\varepsilon}+\beta S_{\varepsilon}$ of the right hand side,

$$
\ln \rho_{\varepsilon} \leq \ln \rho_{\varepsilon}+\beta S_{\varepsilon} \leq \max \left(\ln \rho_{M}^{0}+\beta S_{0}, C_{2}\right) .
$$

Hence the upper bound $\rho_{M}$ for $\rho_{\varepsilon}(t)$.

Thanks to this upper bound, we obtain the lower bound $S_{m}$ on $S_{\varepsilon}(t)$ since, by using the assumption (3) on $Q$, we remark that

$$
\varepsilon \beta \frac{d}{d t} S_{\varepsilon}(t)=Q\left(S_{\varepsilon}(t), \rho_{\varepsilon}(t)\right) \geq Q\left(S_{\varepsilon}(t), \rho_{M}\right)
$$

Then there is a unique value $S_{m}$ such that $Q\left(S_{m}, \rho_{M}\right)=0$, and from the initial conditions (6), we deduce that $S_{m} \leq S_{\varepsilon}(t)$ for $t \geq 0$. 
Next, let us look for the lower bound. It follows, from the integration of (1) as above, that we have

$$
\varepsilon \frac{d}{d t} \ln \rho_{\varepsilon} \geq-K_{2}+\underline{K_{1}} S_{m} .
$$

By subtracting (2) and still using (3), we obtain

$$
\begin{aligned}
\varepsilon \frac{d}{d t}\left(\ln \rho_{\varepsilon}-\beta S_{\varepsilon}\right) & \geq-K_{2}+\underline{K_{1}} S_{m}-Q\left(S_{\varepsilon}, \rho_{\varepsilon}\right) \\
& \geq-K_{2}-Q(0,0)+K_{Q} \rho_{\varepsilon} \\
& \geq-K_{2}-Q(0,0)+K_{Q} e^{\ln \rho_{\varepsilon}-\beta S_{\varepsilon}} e^{\beta S_{m}} .
\end{aligned}
$$

Taking $C_{3}$ the root in $\ln \rho_{\varepsilon}-\beta S_{\varepsilon}$ of the right hand side in (11), we have the lower bound

$$
\rho_{\varepsilon}(t) \geq \min \left(\rho_{m}^{0}, C_{3}\right)
$$

which ends the proof of the Lemma 2.1 .

\subsection{Local $B V$ estimates}

To find local $B V$ bounds for $\rho_{\varepsilon}$ and $S_{\varepsilon}$ which are uniform in $\varepsilon>0$, we apply the method described in [34] that we explain in detail in this section.

Let us first define $J_{\varepsilon}:=\dot{S}_{\varepsilon}$ and $P_{\varepsilon}:=\dot{\rho}_{\varepsilon}$. With these definitions, we have the equations

$$
\varepsilon P_{\varepsilon}=\int n_{\varepsilon} R\left(x, S_{\varepsilon}(t)\right) d x, \quad \varepsilon \beta J_{\varepsilon}=Q\left(\rho_{\varepsilon}(t), S_{\varepsilon}(t)\right) .
$$

Defining $\alpha_{\varepsilon}$ and $\gamma_{\varepsilon}$ as

$$
\alpha_{\varepsilon}(t):=\int n_{\varepsilon} R_{S}\left(\rho_{\varepsilon}(t), S_{\varepsilon}(t)\right) d x \quad \text { and } \quad \gamma_{\varepsilon}(t):=\int n_{\varepsilon} R^{2} d x
$$

we differentiate both equations above, then we obtain the following equations on $J_{\varepsilon}$ and $P_{\varepsilon}$ :

$$
\begin{gathered}
\varepsilon \dot{P}_{\varepsilon}=J_{\varepsilon} \int n_{\varepsilon} R_{S}\left(\rho_{\varepsilon}(t), S_{\varepsilon}(t)\right) d x+\int \partial_{t} n_{\varepsilon} R\left(\rho_{\varepsilon}(t), S_{\varepsilon}(t)\right) d x \\
=\alpha_{\varepsilon}(t) J_{\varepsilon}+\varepsilon \int n_{\varepsilon} \Delta R d x+\frac{1}{\varepsilon} \gamma_{\varepsilon}(t), \\
\varepsilon \beta \dot{J}_{\varepsilon}=Q_{S} J_{\varepsilon}+Q_{\rho} P_{\varepsilon} .
\end{gathered}
$$

However at this stage we cannot obtain directly the $B V$ bounds on $\rho_{\varepsilon}$ and $S_{\varepsilon}$ we expect. Thus we consider a linear combination of $P_{\varepsilon}$ and $J_{\varepsilon}$. Let $\mu_{\varepsilon}(t)$ be a function we will 
determine later. By combining the equalities above, we obtain the following equation on $P_{\varepsilon}+\mu_{\varepsilon} J_{\varepsilon}:$

$$
\begin{aligned}
\varepsilon \frac{d}{d t}\left(P_{\varepsilon}+\beta \mu_{\varepsilon} J_{\varepsilon}\right)= & \alpha_{\varepsilon} J_{\varepsilon}+\varepsilon \int n_{\varepsilon} \Delta R d x+\beta \dot{\mu}_{\varepsilon} J_{\varepsilon}+\mu_{\varepsilon}\left(Q_{S} J_{\varepsilon}+Q_{\rho} P_{\varepsilon}\right)+\frac{1}{\varepsilon} \gamma_{\varepsilon} \\
= & \mu_{\varepsilon} Q_{\rho}\left(P_{\varepsilon}+\beta \mu_{\varepsilon} J_{\varepsilon}\right)+\left(\varepsilon \beta \dot{\mu}_{\varepsilon}-\beta Q_{\rho} \mu_{\varepsilon}^{2}+\mu_{\varepsilon} Q_{S}+\alpha_{\varepsilon}\right) J_{\varepsilon} \\
& +\varepsilon \int n_{\varepsilon} \Delta R d x+\frac{1}{\varepsilon} \gamma_{\varepsilon} .
\end{aligned}
$$

First we prove the following result:

Lemma 3.1. Considering the solution $\mu_{\varepsilon}$ of the differential equation

$$
\varepsilon \beta \dot{\mu}_{\varepsilon}=-\beta\left|Q_{\rho}\right| \mu_{\varepsilon}^{2}+\mu_{\varepsilon}\left|Q_{S}\right|-\alpha_{\varepsilon},
$$

there exist constants $0<\mu_{m}<\mu_{M}$ such that, choosing initially $\mu_{m}<\mu_{\varepsilon}(0)<\mu_{M}$, we have:

$$
\mu_{m} \leq \mu_{\varepsilon}(t) \leq \mu_{M}, \quad \forall t \geq 0 .
$$

Furthermore, we have the following estimate concerning the negative part of the linear combination:

$$
\left(P_{\varepsilon}(t)+\beta \mu(t) J_{\varepsilon}(t)\right)_{-} \leq\left(P_{\varepsilon}(0)+\beta \mu(0) J_{\varepsilon}(0)\right)_{-} e^{\frac{-K_{Q} \mu_{m}}{\varepsilon} t}+\varepsilon C\left(1-e^{\frac{-K_{Q} \mu_{m}}{\varepsilon} t}\right) .
$$

From the estimate of the Lemma 3.1, we can deduce the local $B V$ bounds uniform in $\varepsilon$ we expect. We start with $P_{\varepsilon}$. Adding $\alpha_{\varepsilon} \frac{P_{\varepsilon}}{\beta \mu_{\varepsilon}}$ to 13 and using (5) and Lemma 2.1, we find

$$
\varepsilon \frac{d}{d t} P_{\varepsilon}+\alpha_{\varepsilon} \frac{P_{\varepsilon}}{\beta \mu_{\varepsilon}}=\alpha_{\varepsilon}\left(J_{\varepsilon}+\frac{P_{\varepsilon}}{\beta \mu_{\varepsilon}}\right)+\varepsilon \int n_{\varepsilon} \Delta R d x+\frac{1}{\varepsilon} \gamma_{\varepsilon} \geq-\alpha_{\varepsilon}\left(J_{\varepsilon}+\frac{P_{\varepsilon}}{\beta \mu_{\varepsilon}}\right)_{-}-C \varepsilon .
$$

Notice that $0<\underline{K}_{1} \rho_{\varepsilon}(t) \leq \alpha_{\varepsilon}(t) \leq \bar{K}_{1} \rho_{M}$. By considering the negative parts of $P_{\varepsilon}$ and using (4) and (16), we arrive to the inequality

$$
\begin{aligned}
\varepsilon \frac{d}{d t}\left(P_{\varepsilon}\right)_{-}+\alpha_{\varepsilon} \frac{\left(P_{\varepsilon}\right)_{-}}{\beta \mu_{\varepsilon}} & \leq \alpha_{\varepsilon}\left(J_{\varepsilon}+\frac{P_{\varepsilon}}{\beta \mu_{\varepsilon}}\right)_{-}+C \varepsilon \\
& \leq \alpha_{\varepsilon}\left(P_{\varepsilon}(0)+\beta \mu_{\varepsilon}(0) J_{\varepsilon}(0)\right)_{-} \frac{e^{\frac{-\mu_{m} K_{Q}}{\varepsilon} t}}{\beta \mu_{m}}+\varepsilon \alpha_{\varepsilon} C\left(1-e^{\frac{-\mu_{m} K_{Q}}{\varepsilon} t}\right)+C \varepsilon \\
& \leq \bar{K}_{1} \rho_{M}\left(P_{\varepsilon}(0)+\beta \mu_{\varepsilon}(0) J_{\varepsilon}(0)\right)_{-} \frac{e^{\frac{-\mu_{m} K_{Q}}{\varepsilon} t}}{\beta \mu_{m}}+C \varepsilon
\end{aligned}
$$

With this inequality, the BV bounds follow. Since $\varepsilon P_{\varepsilon}$ is bounded, by integrating the inequality above, we have 


$$
\int_{0}^{T} \alpha_{\varepsilon}(t)\left(P_{\varepsilon}(t)\right)_{-} d t \leq C_{1}(T)+\varepsilon C_{2}(T), \quad \forall T \geq 0 .
$$

Consequently, we obtain

$$
\underline{K}_{1} \int_{0}^{T} \rho_{\varepsilon}\left(\frac{d}{d t} \rho_{\varepsilon}\right)_{-} d x=\frac{K_{1}}{2} \int_{0}^{T}\left(\frac{d}{d t} \rho_{\varepsilon}^{2}\right)_{-} d x \leq \frac{C_{1}(T)+\varepsilon C_{2}(T)}{2}, \quad \forall T \geq 0 .
$$

Since $\rho_{\varepsilon}(t)$ is bounded, we have finally that $\rho_{\varepsilon}^{2}$ has local bounded variations. Therefore up to an extraction, there exists a function $\bar{\rho}$ on $(0, \infty)$ satisfying

$$
\rho_{\varepsilon} \longrightarrow \bar{\rho} \quad \text { in } L_{l o c}^{1}(0, \infty) \text {. }
$$

And since we have the lower bound $\rho_{\varepsilon} \geq \rho_{m}$ by Lemma 1.1, we obtain the bound for the negative part of the derivative of $\rho_{\varepsilon}$ :

$$
\int_{0}^{T}\left(\frac{d}{d t} \rho_{\varepsilon}\right)_{-} d x \leq \frac{C_{1}+C_{2} \varepsilon}{2 \underline{K}_{1} \rho_{m}} .
$$

Finally, it remains to study $S_{\varepsilon}$. To do so, we rewrite (14) as

$$
\varepsilon \beta \frac{d}{d t} J_{\varepsilon}=Q_{S} J_{\varepsilon}+Q_{\rho} P_{\varepsilon}=Q_{S} J_{\varepsilon}+Q_{\rho} \frac{\left(\dot{\rho_{\varepsilon}^{2}}\right)}{2 \rho_{\varepsilon}} .
$$

With our assumptions (3) on the Lipschitz function $Q$, we have

$$
\varepsilon \beta \frac{d}{d t}\left(-J_{\varepsilon}\right)=Q_{S}\left(-J_{\varepsilon}\right)-Q_{\rho} \frac{\left(\dot{\rho_{\varepsilon}^{2}}\right)}{2 \rho} \leq Q_{S}\left(-J_{\varepsilon}\right)+L_{Q} \frac{\left|\left(\dot{\rho_{\varepsilon}^{2}}\right)\right|}{2 \rho_{m}},
$$

and

$$
\varepsilon \beta \frac{d}{d t}\left(J_{\varepsilon}\right)_{-} \leq-K_{Q}\left(J_{\varepsilon}\right)_{-}+L_{Q} \frac{\left|\left(\dot{\rho_{\varepsilon}^{2}}\right)\right|}{2 \rho_{m}} .
$$

The term $\varepsilon J_{\varepsilon}$ is bounded because of our assumptions on $Q$. So, integrating this equation, we have, for $T>0$,

$$
\int_{0}^{T}\left(J_{\varepsilon}\right)_{-} \leq C+\frac{L_{Q}}{2 \rho_{m} K_{Q}} \int_{0}^{T}\left|\left(\dot{\rho_{\varepsilon}^{2}}\right)\right|
$$

and we deduce that $\int_{0}^{T}\left(J_{\varepsilon}\right)_{-}$is uniformly bounded from our previous results on $\rho_{\varepsilon}^{2}$. And then, since $S_{\varepsilon}$ is uniformly bounded, we conclude that there exists a function $\bar{S}(t)$ such that, after extraction of a subsequence,

$$
S_{\varepsilon} \longrightarrow \bar{S} \quad \text { in } L_{l o c}^{1}(0, \infty) \quad \text { and } \quad Q\left(S_{\varepsilon}, \rho_{\varepsilon}\right) \underset{\varepsilon \rightarrow 0}{\longrightarrow} Q(\bar{S}, \bar{\rho}) \quad \text { a.e. }
$$

To conclude, it follows that $\varepsilon \frac{d}{d t} S_{\varepsilon}$ converges in measure to 0 as $\varepsilon$ vanishes and thus, $Q(\bar{S}, \bar{\rho})=0$. 


\subsection{Proof of Lemma 3.1}

Our goal is to choose a function $\mu_{\varepsilon}(t)$ which solves the differential equation

$$
\varepsilon \beta \dot{\mu}_{\varepsilon}=-\beta\left|Q_{\rho}\right| \mu_{\varepsilon}^{2}+\mu_{\varepsilon}\left|Q_{S}\right|-\alpha_{\varepsilon}
$$

We use the same argument as in [34]. Therefore we concentrate on the main ideas.

Note that, because the solution might blow up to $-\infty$ in finite time, we need to prove that solutions of $(22)$ which remain strictly positive for all times. To do so, we first notice that the zeroes of $-\beta\left|Q_{\rho}\right| \mu_{\varepsilon}^{2}+\mu_{\varepsilon}\left|Q_{S}\right|-\alpha_{\varepsilon}$ are

$$
\mu_{\varepsilon, \pm}(t):=\frac{1}{2 \beta\left|Q_{\rho}\right|}\left(\left|Q_{S}\right| \pm \sqrt{Q_{S}^{2}-4 \alpha_{\varepsilon} \beta\left|Q_{\rho}\right|}\right)
$$

and from the smallness condition (7), both zeros are positive.

We need to find two constants $0<\mu_{m}<\mu_{M}$ such that, choosing initially $\mu_{m}<\mu_{\varepsilon}(0)<$ $\mu_{M}$, then we have for all times

$$
0<\mu_{m} \leq \mu_{\varepsilon}(t) \leq \mu_{M}
$$

This condition is satisfied with the following constants

$$
\mu_{M}:=\frac{1}{\beta} \max _{\substack{\rho_{m} \leq \rho \leq \rho_{M} \\ S_{m} \leq S \leq S_{0}}} \frac{\left|Q_{S}\right|}{\left|Q_{\rho}\right|}
$$

and $\mu_{m}$ defined as

$$
\max _{t} \mu_{\varepsilon,-}(t) \leq \mu_{m}:=\min _{t} \mu_{\varepsilon,+}(t)
$$

which defines a positive constant because of the smallness condition for $\beta$ (7).

Coming back to equation 15, , we arrive to

$$
\varepsilon \frac{d}{d t}\left(P_{\varepsilon}+\beta \mu J_{\varepsilon}\right) \geq-\mu\left|Q_{\rho}\right|\left(P_{\varepsilon}+\beta \mu J_{\varepsilon}\right)+\varepsilon \int n_{\varepsilon} \Delta R d x \geq-\mu\left|Q_{\rho}\right|\left(P_{\varepsilon}+\beta \mu J_{\varepsilon}\right)-\varepsilon C,
$$

and we conclude that, for all $t \geq 0$,

$$
\left(P_{\varepsilon}(t)+\beta \mu(t) J_{\varepsilon}(t)\right)_{-} \leq\left(P_{\varepsilon}(0)+\beta \mu(0) J_{\varepsilon}(0)\right)_{-} e^{\frac{-K_{Q} \mu_{m}}{\varepsilon} t}+\varepsilon C\left(1-e^{\frac{-K_{Q} \mu_{m}}{\varepsilon} t}\right)
$$

which concludes the proof of the Lemma 3.1. 


\section{Concentration and constrained Hamilton-Jacobi equation}

In order to prove the concentration of $n_{\varepsilon}$ in a sum of Dirac masses as $\varepsilon$ vanishes, we perform the change of unknown $n_{\varepsilon}(t, x)=e^{u_{\varepsilon}(t, x) / \varepsilon}$ and we study the regularity properties of $u_{\varepsilon}(t, x)$. With the definition of $u_{\varepsilon}$, we obtain the following equation which is equivalent to (1):

$$
\left\{\begin{array}{l}
\partial_{t} u_{\varepsilon}(t, x)=\left|\nabla u_{\varepsilon}\right|^{2}+R\left(x, S_{\varepsilon}(t)\right)+\varepsilon \Delta u_{\varepsilon}, \\
u_{\varepsilon}(t=0, x)=u_{\varepsilon}^{0}(x):=\varepsilon \ln n_{\varepsilon}^{0}
\end{array}\right.
$$

We complete assumption (6) on the initial data with

$$
u_{\varepsilon}^{0}(x) \leq A-K_{2} \sqrt{1+|x|^{2}}, \quad\left\|\nabla u_{\varepsilon}^{0}\right\| \leq B, \quad \forall x \in \mathbb{R}^{d},
$$

with $A, B>0$.

We prove in this section the following result

Theorem 4.1. Under the assumptions (3)-(7) and (28), then after extraction of a subsequence $\left(u_{\varepsilon}\right)_{\varepsilon}$ converges locally uniformly to a Lipschitz continuous viscosity solution $u$ to the constrained Hamilton-Jacobi equation

$$
\left\{\begin{array}{l}
\partial_{t} u(t, x)=|\nabla u|^{2}+R(x, \bar{S}(t)) \\
\max _{x \in \mathbb{R}^{d}} u(t, x)=0, \quad \forall t \geq 0
\end{array}\right.
$$

In the simple case when dimension $d$ is equal to 1 and when $R(x, S)$ is monotonic in $x$ for all $S, n$ concentrates in one single point.

We first prove that $u_{\varepsilon}$ is equi-bounded, then the equi-continuity, and finally we explain how to pass to the limit in 27 .

\subsection{An upper bound for $u_{\varepsilon}$}

We first set the upper bound for $u_{\varepsilon}$. Let $T>0$ be given. Defining $\bar{u}(t, x)=A+C t-$ $K_{2} \sqrt{1+|x|^{2}}$ with $C=K_{2}\left(1+K_{2}\right)$, we have

$$
\partial_{t} \bar{u}-\varepsilon \Delta \bar{u}-|\nabla \bar{u}|^{2}-R\left(x, S_{\varepsilon}(t)\right) \geq C+\varepsilon K_{2} \frac{d-1}{\sqrt{1+|x|^{2}}}-K_{2}^{2}-K_{2} \geq 0 .
$$

Since $\bar{u}(0, x) \geq u_{\varepsilon}^{0}(x)$ from initial data $(28)$, we conclude that $\bar{u}$ is a super-solution and $u_{\varepsilon}(t, x) \leq A+C T-K_{2} \sqrt{1+|x|^{2}}$, for all $t \in[0, T]$. 


\subsection{Lipschitz bound in space}

We first prove that $u_{\varepsilon}$ is uniformly Lipschitz continuous in space on $[0, T] \times \mathbb{R}^{d}$. We define for $h$ small $w_{\varepsilon}(t, x)=u_{\varepsilon}(t, x+h)-u_{\varepsilon}(t, x)$. Since the initial condition $u_{\varepsilon}^{0}$ are uniformly continuous, given $\delta>0$, for $h$ small enough, we have $\left|w_{\varepsilon}(0, x)\right|<\frac{\delta}{2}$. From (27), we arrive to

$$
\begin{aligned}
\partial_{t} w_{\varepsilon}(t, x)-\varepsilon \Delta w_{\varepsilon}(t, x)- & \left(\nabla u_{\varepsilon}(t, x+h)+\nabla u_{\varepsilon}(t, x)\right) \cdot \nabla w_{\varepsilon}(t, x) \\
= & R\left(x+h, S_{\varepsilon}(t)\right)-R\left(x, S_{\varepsilon}(t)\right) \leq K_{2} h .
\end{aligned}
$$

Thus by the maximum principle we deduce that

$$
\left|w_{\varepsilon}(t, x)\right| \leq\left|\max _{\mathbb{R}^{d}} w_{\varepsilon}(0, x)\right|+K_{2}|h| t \leq\left(\left\|\nabla u_{\varepsilon}^{0}\right\|_{L^{\infty}\left(\mathbb{R}^{d}\right)}+K_{2} t\right)|h|
$$

We conclude that $u_{\varepsilon}$ is uniformly Lipschitz in space on $[0, T] \times \mathbb{R}^{d}$ and set

$$
L(t)=\sup _{\varepsilon \leq \varepsilon_{0}, 0 \leq s \leq t, x \in \mathbb{R}^{d}}\left\|\nabla u_{\varepsilon}(t, x)\right\|_{L^{\infty}} .
$$

\subsection{Local bounds for $u_{\varepsilon}$}

We already know from the first step that $u_{\varepsilon}$ is locally bounded from above. We show that it is also bounded from below on compact subsets of $[0, \infty) \times \mathbb{R}^{d}$. Let $0<T$ and $r>0$. For all $t \in[0, T]$ and $x \in B(0, r)$, we recall that $u_{\varepsilon}(t, x) \leq A+C T-K_{2} \sqrt{1+|x|^{2}}$ and thus

$$
\int_{|x|>r} e^{\frac{u_{\varepsilon}}{\varepsilon}} d x<\int_{|x|>r} e^{\frac{A+C T-K_{2}|x|}{\varepsilon}}<\frac{\rho_{m}}{2},
$$

for $0<\varepsilon<\varepsilon_{0}, \varepsilon_{0}$ small enough and $r$ large enough. We also have from Lemma 3.1 that $\rho_{\varepsilon} \geq \rho_{m}$, then for $0<\varepsilon<\varepsilon_{0}$ and $r$ large enough, we obtain

$$
\frac{\rho_{m}}{2}<\int_{|x| \leq r} e^{\frac{u_{\varepsilon}}{\varepsilon}} \leq B_{r} e^{\max _{r} \frac{u_{\varepsilon}}{\varepsilon}}
$$

This implies

$$
\max _{B_{r}} u_{\varepsilon} \geq \varepsilon \ln \frac{\rho_{m}}{2\left|B_{r}\right|} .
$$

Using the Lipschitz bound (31) we obtain

$$
u_{\varepsilon}(t, x)>\varepsilon \ln \frac{\rho_{m}}{2\left|B_{r}\right|}-2 L(t) r, \quad \forall x \in \mathbb{R}^{d} .
$$

Hence we have the local lower bound on $u_{\varepsilon}$. 


\subsection{The equi-continuity in time}

For given $T, \eta$ and $r>0$, we fix $(s, x) \in\left[0, T\left[\times B\left(0, \frac{r}{2}\right)\right.\right.$ and define

$$
\xi_{\varepsilon}(t, y)=u_{\varepsilon}(s, x)+\eta+E|y-x|^{2}+D(t-s), \quad \text { for }(t, y) \in[s, T] \times B(0, r),
$$

where $E$ and $D$ are constants to be determined. We prove in this section the uniform continuity in time. The idea of the proof is to find constants $E$ and $D$ large enough such that, for any $x \in R\left(0, \frac{r}{2}\right)$, and for all $\varepsilon<\varepsilon_{0}$

$$
u_{\varepsilon}(t, y) \leq \xi_{\varepsilon}(t, y)=u_{\varepsilon}(s, x)+\eta+E|y-x|^{2}+D(t-s), \quad \forall(t, y) \in[0, T] \times B(0, r),
$$

and

$$
u_{\varepsilon}(t, y) \geq \phi_{\varepsilon}(t, y):=u_{\varepsilon}(s, x)-\eta-E|y-x|^{2}-D(t-s), \quad \forall(t, y) \in[0, T] \times B(0, r) .
$$

Then by taking $y=x$, we have the uniform continuity in time on compact subsets of $[0, \infty) \times \mathbb{R}^{d}$. We prove here inequality $(32)$, the proof of $(33)$ is analogous.

First we prove that $\xi_{\varepsilon}(t, y)>u_{\varepsilon}(t, y)$ on $[s, T] \times \partial B(0, r)$, for all $\eta, D$ and $x \in B\left(0, \frac{r}{2}\right)$. Since $u_{\varepsilon}$ are locally uniformly bounded according to Sections 4.1 and 4.3 , by taking $\mathrm{E}$ large enough such that

$$
E \geq \frac{8\left\|u_{\varepsilon}\right\|_{L^{\infty}([0, T] \times B(0, r))}}{r^{2}}
$$

we obtain

$$
\begin{aligned}
\xi_{\varepsilon}(t, y) & \geq u_{\varepsilon}(t, x)+\eta+2\left\|u_{\varepsilon}\right\|_{L^{\infty}([0, T] \times B(0, r))}+D(t-s) \\
& \geq\left\|u_{\varepsilon}\right\|_{L^{\infty}([0, T] \times B(0, r))} \\
& \geq u_{\varepsilon}(t, y) .
\end{aligned}
$$

Next we prove that, for $E$ large enough, $\xi_{\varepsilon}(s, y) \geq u_{\varepsilon}(s, y)$ for all $y \in B(0, r)$. We argue by contradiction. Assume that there exists $\eta>0$ such that for all constants $E>0$ there exists $y_{E} \in B(0, r)$ such that

$$
u_{\varepsilon}\left(s, y_{E}\right)-u_{\varepsilon}(s, x)>\eta+E\left|y_{E}-x\right|^{2} .
$$

This implies

$$
\left|y_{E}-x\right| \geq \sqrt{\frac{2 M}{E}}
$$

where $\mathrm{M}$ is a uniform upper bound for $\left\|u_{\varepsilon}\right\|_{L^{\infty}([0, T] \times B(0, r))}$. For $E \rightarrow \infty$, we have that $\left|y_{E}-x\right| \rightarrow 0$. Since $u_{\varepsilon}$ are uniformly continuous in space, this is a contradiction.

Finally, from assumption (5), if $D$ is large enough, $\xi_{\varepsilon}$ is a super-solution to (29) in $[s, T] \times B(0, r)$,

$$
u_{\varepsilon}(t, y) \leq u_{\varepsilon}(s, x)+\eta+E|y-x|^{2}+D(t-s), \quad \forall(t, y) \in[0, T] \times B(0, r) .
$$

With the proof of (33) which is similar, we deduce that the sequence $u_{\varepsilon}$ is uniformly continuous in time on compact subsets of $[0, \infty) \times \mathbb{R}^{d}$. 


\subsection{Passing to the limit}

We proceed as in [5] to prove the convergence of (27) to (29) as $\varepsilon$ goes to 0. Considering the regularity results above, the point at this step is to pass to the limit in the term $R\left(x, S_{\varepsilon}\right)$. To avoid the complications of the discontinuity, we define

$$
\phi_{\varepsilon}(t, x):=u_{\varepsilon}(t, x)-\int_{0}^{t} R\left(x, S_{\varepsilon}(s)\right) d s,
$$

and it follows that $\phi_{\varepsilon}$ satisfies the equation:

$$
\begin{aligned}
\partial_{t} \phi_{\varepsilon}(t, x)-\varepsilon \Delta \phi_{\varepsilon}(t, x) & -\left|\nabla \phi_{\varepsilon}(t, x)\right|^{2}-2 \nabla \phi_{\varepsilon}(t, x) \cdot \int_{0}^{t} \nabla R\left(x, S_{\varepsilon}(s)\right) d s \\
& =\varepsilon \int_{0}^{t} \Delta R\left(x, S_{\varepsilon}(s)\right) d s+\left|\int_{0}^{t} \nabla R\left(x, S_{\varepsilon}(s)\right) d s\right|^{2} .
\end{aligned}
$$

As $S_{\varepsilon}(t)$ converges to $\bar{S}(t)$ for all $t \geq 0$ and $R(x, I)$ is a Lipschitz continuous function, we have

$$
\begin{aligned}
\lim _{\varepsilon \rightarrow 0} \int_{0}^{t} R\left(x, S_{\varepsilon}(s)\right) d s & =\int_{0}^{t} R(x, \bar{S}(s)) d s, \\
\lim _{\varepsilon \rightarrow 0} \int_{0}^{t} \nabla R\left(x, S_{\varepsilon}(s)\right) d s & =\int_{0}^{t} \nabla R(x, \bar{S}(s)) d s, \\
\lim _{\varepsilon \rightarrow 0} \int_{0}^{t} \Delta R\left(x, S_{\varepsilon}(s)\right) d s & =\int_{0}^{t} \Delta R(x, \bar{S}(s)) d s,
\end{aligned}
$$

for all $t \geq 0$. Furthermore the limit functions $\int_{0}^{t} R(x, \bar{S}(s)) d s, \int_{0}^{t} \nabla R(x, \bar{S}(s)) d s$ and $\int_{0}^{t} \Delta R(x, \bar{S}(s)) d s$ are locally uniformly continuous.

After extraction of a subsequence by the Arzela-Ascoli Theorem, $u_{\varepsilon}(t, x)$ converges locally uniformly to the continuous function $u(t, x)$ as $\varepsilon$ vanishes. Consequently $\phi_{\varepsilon}(t, x)$ converges locally uniformly to the continuous function $\phi(t, x)=u(t, x)-\int_{0}^{t} R(x, \bar{S}(s)) d s$ and $\phi$ is a viscosity solution to the equation

$$
\partial_{t} \phi(t, x)-|\nabla \phi(t, x)|^{2}-2 \nabla \phi(t, x) . \int_{0}^{t} \nabla R(x, \bar{S}(s)) d s=\left|\int_{0}^{t} \nabla R(x, \bar{S}(s)) d s\right|^{2} .
$$

Then $u$ is a solution to the following equation in the viscosity sense

$$
\partial_{t} u(t, x)=|\nabla u|^{2}+R(x, \bar{S}(t)) .
$$

It remains to prove that $\max _{x \in \mathbb{R}^{d}} u(t, x)=0$ for all $t \geq 0$. We argue by contradiction. Assume that there exists $a>0$ such that for some $t>0$ and $x \in \mathbb{R}^{d}$ we have $0<a \leq u(t, x)$. It follows that, from the continuity of $u, u(t, y) \geq \frac{a}{2}$ on $B(x, r)$ for some $r>0$, and then $n_{\varepsilon}(t, y) \rightarrow \infty$ as $\varepsilon$ goes to 0 , which is a contradiction to the statements of 
Lemma 2.1. Thus we have $\max _{x \in \mathbb{R}^{d}} u(t, x) \leq 0$ for all $t \geq 0$.

From the section 4.3, we have for $0<\varepsilon<\varepsilon_{0}$ and for some $r>0$ large enough

$$
\lim _{\varepsilon \rightarrow 0} \int_{|x| \leq r} n_{\varepsilon}(t, x) d x>\frac{\rho_{m}}{2}, \quad t \geq 0 .
$$

Furthermore, recall that, from section 4.1, we have

$$
u_{\varepsilon}(t, x) \leq A+C t-K_{2} \sqrt{1+|x|^{2}} \leq A+C t-K_{2}|x|, \quad \forall t \geq 0, x \in \mathbb{R}^{d} .
$$

Then it follows that, for $r$ large enough

$$
\lim _{\varepsilon \rightarrow 0} \int_{|x| \geq r} n_{\varepsilon}(t, x) d x \leq \lim _{\varepsilon \rightarrow 0} e^{\frac{A+C t-K_{2}|x|}{\varepsilon}} d x=0 .
$$

We argue by contradiction again. Assume that $u(t, x)<0$ for all $t \geq 0$ and $|x|<r$. It implies that $\lim _{\varepsilon \rightarrow 0} n_{\varepsilon}(t, x)=0$ and thus $\lim _{\varepsilon \rightarrow 0} \int_{|x|<r} n_{\varepsilon}(t, x) d x=0$. This is a contradiction with (36) and it follows that $\max _{x \in \mathbb{R}^{d}} u(t, x)=0$ for all $t \geq 0$.

It is an open problem to know if the full sequence $u_{\varepsilon}$ converges and it is equivalent to the question of uniqueness of the solution to the Hamilton-Jacobi equation. We will consider in section 5 a special case where uniqueness holds.

In the next section we derive some properties of the concentration points that also hold in the concavity framework (section 6) and will be useful in what follows.

\subsection{Properties of the concentration points}

We prove in the rest of this section the following theorem

Theorem 4.2. Let assumption (5) hold. For any $u^{0} \in W^{1, \infty}\left(\mathbb{R}^{d}\right)$, the solution to (29) is semi-convex in $x$ for any $t>0$, i.e. there exists a $C(t)$ such that, for any unit vector $\xi \in \mathbb{R}^{d}$, we have the following inequality

$$
\frac{\partial^{2}}{\partial \xi^{2}} u \geq-C
$$

Consequently, $u(t, \cdot)$ is differentiable in $x$ at maximum points and we have

$$
\nabla u(t, \bar{x}(t))=0
$$

where $\bar{x}(t)$ is a maximum point of $u(t, \cdot)$.

Furthermore, for all Lebesgue points of $\bar{S}$ we have

$$
R(\bar{x}(t), \bar{S}(t))=0
$$


First step: the semi-convexity. To increase readability we use the notation $u_{\xi}:=\frac{\partial u_{\varepsilon}}{\partial \xi}, u_{\xi \xi}:=$ $\frac{\partial^{2} u_{\varepsilon}}{\partial \xi^{2}}$ for a unit vector $\xi$. We obtain from equation (27)

$$
\frac{\partial}{\partial t} u_{\xi}=2 \nabla u_{\varepsilon} \cdot \nabla u_{\xi}+R_{\xi}\left(x, S_{\varepsilon}(t)\right)+\varepsilon \Delta u_{\xi},
$$

and

$$
\frac{\partial}{\partial t} u_{\xi \xi}=2 \nabla u_{\varepsilon} \cdot \nabla u_{\xi \xi}+2\left|\nabla u_{\xi}\right|^{2}+R_{\xi \xi}\left(x, S_{\varepsilon}(t)\right)+\varepsilon \Delta u_{\xi \xi} .
$$

Notice that $\left|\nabla u_{\xi}\right| \geq\left|u_{\xi \xi}\right|$ because $u_{\xi \xi}=\nabla u_{\xi} \cdot \xi$. Therefore the function $w:=u_{\xi \xi}$ satisfies

$$
\frac{\partial}{\partial t} w \geq 2 \nabla u_{\varepsilon} \cdot \nabla w+2 w^{2}-K_{2}+\varepsilon \Delta w,
$$

from the assumption (5). The semi-convexity follows from the comparison principle with the subsolution given by the solution to the ODE $\dot{y}=2 y^{2}-K_{2}, y(0)=-\infty$.

Second step: $\nabla u(t, \bar{x}(t))=0$. The semi-convexity implies that $u$ is differentiable at its maximum points. Therefore we have for $t>0$

$$
\nabla u(t, \bar{x}(t))=0
$$

Moreover, we also have the property that, for any sequence $\left(t_{k}, x_{k}\right)$ of $x$-differentiability point of $u$ which converges to $(t, \bar{x}(t))$, we have

$$
\nabla u\left(t_{k}, x_{k}\right) \rightarrow 0 \quad \text { as } \quad k \rightarrow \infty
$$

In fact, we deduce that, for $h, r>0, h, r \rightarrow 0$

$$
\frac{1}{r h} \int_{t}^{t+h} \int_{\bar{x}(t)-r}^{\bar{x}(t)+r}|\nabla u(s, y)|^{2} d s d y \rightarrow 0
$$

and

$$
\frac{1}{r h} \int_{t-h}^{t} \int_{\bar{x}(t)-r}^{\bar{x}(t)+r}|\nabla u(s, y)|^{2} d s d y \rightarrow 0 .
$$

We obtain these convergence results by applying Lebesgue's dominated convergence Theorem to the integral

$$
\int_{0}^{1} \int_{-1}^{1}|\nabla u(t+h \tau, x(t)+r \sigma)|^{2} d \tau d \sigma
$$

given by a change of variable, combined with the local Lipschitz continuity of $u$.

Third Step: Proof of $R(\bar{x}(t), \bar{S}(t))=0$. We first integrate the equation on rectangles $(t, t+h) \times(\bar{x}(t)-r, \bar{x}(t)+r)$. We obtain 
$\int_{\bar{x}(t)-r}^{\bar{x}(t)+r}[u(t+h, y)-u(t, y)] d y=\int_{t}^{t+h} \int_{\bar{x}(t)-r}^{\bar{x}(t)+r} R(y, \bar{S}(s)) d s d y+\int_{t}^{t+h} \int_{\bar{x}(t)-r}^{\bar{x}(t)+r}|\nabla u(s, y)|^{2} d s d y$.

By the semi-convexity, we have

$$
0 \geq u(t, y) \geq u(t, \bar{x}(t))-C|y-\bar{x}(t)|^{2}=O\left(r^{2}\right),
$$

and also $u(t+h, y) \leq 0$. We deduce

$$
\frac{1}{r h} \int_{t}^{t+h} \int_{\bar{x}(t)-r}^{\bar{x}(t)+r} R(y, \bar{S}(s)) d s d y+\frac{1}{r h} \int_{t}^{t+h} \int_{\bar{x}(t)-r}^{\bar{x}(t)+r}|\nabla u(s, y)|^{2} d s d y \leq \frac{1}{r h} O\left(r^{2}\right) .
$$

Therefore we obtain

$$
\frac{1}{r h} \int_{t}^{t+h} \int_{\bar{x}(t)-r}^{\bar{x}(t)+r} R(y, \bar{S}(s)) d s d y \leq \frac{1}{r h} O\left(r^{2}\right) .
$$

We conclude that at any Lebesgue point of $\bar{S}$ we have

$$
R(\bar{x}(t), \bar{S}(t)) \leq 0
$$

Next, we prove the opposite inequality. By integrating on the rectangle $(t-h, t) \times$ $(\bar{x}(t)-r, \bar{x}(t)+r)$.

$$
\int_{\bar{x}(t)-r}^{\bar{x}(t)+r}(u(t, y)-u(t-h, y)) d y \geq \int_{\bar{x}(t)-r}^{\bar{x}(t)+r} u(t, y) d y
$$

and

$$
\frac{1}{r h} \int_{t-h}^{t} \int_{\bar{x}(t)-r}^{\bar{x}(t)+r} R(y, \bar{S}(s)) d s d y+\frac{1}{r h} \int_{t-h}^{t} \int_{\bar{x}(t)-r}^{\bar{x}(t)+r}|\nabla u(s, y)|^{2} d s d y \geq \frac{O(r)}{h} .
$$

Hence, we have that, at any Lebesgue point of $\bar{S}$,

$$
R(\bar{x}(t), \bar{S}(t)) \leq 0
$$

Hence the statement of Theorem 4.2.

\section{The monomorphic case in dimension $d=1$}

In the case when dimension $d$ equals 1 and $R(x, S)$ is monotonic in $x$ for each $S$, we have the expected convergence toward a single Dirac mass under the additional assumption (which holds for instance when $R$ is monotonic in $x$ )

$$
\forall S_{m}<S<S_{0}, \text { there is a unique } X(S) \in \mathbb{R} \text { such that } R(X(S), S)=0 .
$$


Theorem 5.1. Assume (3)-(7), that $u_{\varepsilon}^{0}$ are uniformly continuous in $\mathbb{R}^{d}$ and (39). Then, the solution $n_{\varepsilon}$ to (1), still after extraction of a subsequence, converges in the weak sense of measures

$$
n_{\varepsilon_{k}}(t, x) \longrightarrow n(t, x):=\bar{\rho}(t) \delta(x-\bar{x}(t))
$$

and we also obtain the relations

$$
\bar{x}(t)=X(\bar{S}(t)), \quad R(\bar{x}(t), \bar{S}(t))=0 \quad \text { a.e. }
$$

Moreover, the full sequence $n_{\varepsilon}$ converges when $R$ has one of the following form, for some functions $b>0, d>0, F>0$,

$$
R(x, S)=b(x)-d(x) F(S) \quad \text { with } F^{\prime}(S)<0,
$$

or

$$
R(x, S)=b(x) F(S)-d(x) \quad \text { with } F^{\prime}(S)>0 .
$$

We do not prove this result in detail. It is a consequence of the following observation. As the measure $n$ defined in (40) satisfies the condition supp $n(t, \cdot) \subset\{u(t, \cdot)\}$ from the properties obtained in the previous section (see details in [7, 5]), $n$ is monomorphic. Indeed, from the condition $(39)$ the set $\{u(t, \cdot)\}$ is reduced to an isolated point for all $t \geq 0$. The uniqueness of the solution when $R$ is written as (41) or (42) is entirely explained in [7]. The idea of the proof is to consider for instance the function

$$
\phi(t, x)=u(t, x)-b(x) \int_{0}^{t} F(S(\sigma)) d \sigma,
$$

and, by noticing that $\phi$ satisfies the equation

$$
\partial_{t} \phi(t, x)=-d(x)+\left|\nabla(\phi(t, x)+b(x)) \int_{0}^{t} F(S(\sigma)) d \sigma\right|,
$$

to derive an estimate on the derivative of the difference between two different solutions $\phi_{1}$ and $\phi_{2}$ with the same initial data. By considering the different quantities at the maximum points of $u(t, \cdot)$, it comes that there exists a constant $C>0$ such that

$$
\frac{d}{d t}\left\|\phi_{1}-\phi_{2}\right\|_{\infty} \leq C\left\|\phi_{1}-\phi_{2}\right\|_{\infty}
$$

and the uniqueness follows.

\section{The concavity framework in $\mathbb{R}^{d}$}

In this section we are going to assume more regularity in order to prove the convergence of $n_{\varepsilon}$ to a Dirac mass in the sense of measure. The specific feature of this framework is that uniform concavity of the growth rate and initial data induce uniform concavity of 
the solutions $u_{\varepsilon}$ to the Hamilton-Jacobi equations, which implies that $u_{\varepsilon}$ has only one maximum point. The main technical difficulty is that uniform bounds are not possible because of the quadratic growth at infinity. Therefore, following the work [33], we start with assumptions on $R \in C^{2}$ :

$$
\begin{gathered}
\max _{x \in \mathbb{R}^{d}} R\left(x, S_{m}\right)=0=R\left(0, S_{m}\right), \\
-\underline{K}_{2}|x|^{2} \leq R(x, S) \leq \bar{K}_{0}-\bar{K}_{2}|x|^{2}, \\
0<\underline{K}_{1} \leq R_{S}(x, S) \leq \bar{K}_{1}, \\
-2 \underline{K}_{2} \leq D^{2} R(x, S) \leq-2 \bar{K}_{2} .
\end{gathered}
$$

We also need the uniform concavity of the initial data

$$
\begin{gathered}
n_{\varepsilon}^{0}=e^{\frac{u_{\varepsilon}^{0}}{\varepsilon}}, \\
-\underline{L}_{0}-\underline{L}_{1}|x|^{2} \leq u_{\varepsilon}^{0} \leq \bar{L}_{0}-\bar{L}_{1}|x|^{2}, \\
-2 \underline{L}_{1} \leq D^{2} u_{\varepsilon}^{0} \leq-2 \bar{L}_{1},
\end{gathered}
$$

and we add some compatibility conditions

$$
4 \bar{L}_{1}^{2} \leq \bar{K}_{2} \leq \underline{K}_{2} \leq 4 \underline{L}_{1}^{2}
$$

For this section, we will need

$$
\begin{gathered}
D^{3} R(\cdot, S) \in L^{\infty}\left(\mathbb{R}^{d}\right), \\
D^{3} u_{\varepsilon}^{0} \in L^{\infty}\left(\mathbb{R}^{d}\right) \quad \text { uniformly in } \varepsilon, \\
n_{\varepsilon}^{0}(x) \longrightarrow \bar{\rho}^{0} \delta\left(x-\bar{x}^{0}\right) \quad \text { weakly in the sense of measures. }
\end{gathered}
$$

We keep the same assumptions on $Q$ and $S_{\varepsilon}$ as in the previous section. Next we are going to prove the following result:

Theorem 6.1. Under assumptions (44)-(50) and the assumptions on $Q, \rho_{\varepsilon}$ and $S_{\varepsilon}$ have locally bounded total variations uniformly in $\varepsilon$. Therefore there exist functions $\bar{\rho}$ and $\bar{S}$ such that, after extraction of a subsequence, we have

$$
S_{\varepsilon_{k}}(t) \underset{\varepsilon_{k} \rightarrow 0}{\longrightarrow} \bar{S}(t) \quad \text { and } \quad \rho_{\varepsilon_{k}}(t) \underset{\varepsilon_{k} \rightarrow 0}{\longrightarrow} \bar{\rho}(t), \quad \text { a.e. }
$$

Furthermore we have weakly in the sense of measures for a subsequence $n_{\varepsilon}$

$$
n_{\varepsilon}(t, x) \underset{\varepsilon \rightarrow 0}{\longrightarrow} \bar{\rho}(t) \delta(x-\bar{x}(t))
$$

and the pair $(\bar{x}(t), \bar{S}(t))$ also satisfies

$$
R(\bar{x}(t), \bar{S}(t))=0, \quad \text { a.e. }
$$


As a first step, we will give estimates on $u_{\varepsilon}$. Next, we will adapt the proof of the section 3 to give $B V$ estimates on $\rho_{\varepsilon}$ and $S_{\varepsilon}$ and then pass to the limit as $\varepsilon$ goes to 0 . Finally we prove the following theorems:

Theorem 6.2. Assuming 433)-(53), $\bar{x}(t)$ is a $W^{1, \infty}\left(\mathbb{R}_{+}, \mathbb{R}^{d}\right)$-function and its dynamics is described by the equation

$$
\dot{\bar{x}}(t)=\left(-D^{2} u(t, \bar{x}(t))\right)^{-1} \cdot \nabla_{x} R(\bar{x}(t), \bar{S}(t)), \quad \bar{x}(0)=\bar{x}^{0}
$$

with $u(t, x)$ given below in (71) and $\bar{x}^{0}$ in $(53)$. Furthermore, $\bar{S}(t)$ is a $W^{1, \infty}\left(\mathbb{R}_{+}\right)$-function. From this equation, it follows that $\bar{S}(t)$ is a decreasing function and

$$
\bar{S}(t) \underset{t \rightarrow \infty}{\longrightarrow} S_{m}, \quad \bar{x}(t) \underset{t \rightarrow \infty}{\longrightarrow} 0 .
$$

\subsection{Uniform concavity of $u_{\varepsilon}$}

Again we use the Hopf-Cole transformation defining $u_{\varepsilon}=\varepsilon \ln n_{\varepsilon}$ and we obtain the same equation as in Section 4

$$
\left\{\begin{array}{l}
\partial_{t} u_{\varepsilon}(t, x)=\left|\nabla u_{\varepsilon}\right|^{2}+R\left(x, S_{\varepsilon}(t)\right)+\varepsilon \Delta u_{\varepsilon} \\
u_{\varepsilon}(t=0, x)=u_{\varepsilon}^{0}(x):=\varepsilon \ln n_{\varepsilon}^{0}
\end{array}\right.
$$

We focus now on the study of the properties of the sequence $u_{\varepsilon}$.

We first prove the following lemma

Lemma 6.3. Under assumptions (44) and (50), we have for $t \geq 0$ and for $x \in \mathbb{R}^{d}$

$$
-\underline{L}_{0}-\underline{L}_{1}|x|^{2}-\varepsilon\left(2 d \underline{L}_{1}\right) t \leq u_{\varepsilon}(t, x) \leq \bar{L}_{0}-\bar{L}_{1}|x|^{2}+\left(\bar{K}_{0}+2 d \varepsilon \bar{L}_{1}\right) t .
$$

Proof. First we achieve an upper bound for $u_{\varepsilon}$. By defining $\bar{u}_{\varepsilon}(t, x):=\bar{L}_{0}-\bar{L}_{1}|x|^{2}+C_{0}(\varepsilon) t$ with $C_{0}(\varepsilon):=\bar{K}_{0}+2 d \varepsilon \bar{L}_{1}$, we obtain from assumptions (44), (48) and (50) that $\bar{u}_{\varepsilon}(t=$ $0) \geq u_{\varepsilon}^{0}$ and

$$
\partial_{t} \bar{u}_{\varepsilon}-\left|\nabla \bar{u}_{\varepsilon}\right|^{2}-R\left(x, I_{\varepsilon}\right)-\varepsilon \Delta \bar{u}_{\varepsilon} \geq C_{0}(\varepsilon)-4 \bar{L}_{1}^{2}|x|^{2}-\bar{K}_{0}+\bar{K}_{2}|x|^{2}-2 d \varepsilon \bar{L}_{1} \geq 0 .
$$

Then by a comparison principle, we conclude that $u_{\varepsilon}(t, x) \leq \bar{L}_{0}-\bar{L}_{1}|x|^{2}+\left(\bar{K}_{0}+2 d \varepsilon \bar{L}_{1}\right) t$ for all $t \geq 0$ and $x \in \mathbb{R}^{d}$.

Next for the lower bound, we define $\underline{u}_{\varepsilon}(t, x):=-\underline{L}_{0}-\underline{L}_{1}|x|^{2}-\varepsilon C_{1} t$ with $C_{1}:=2 d \underline{L}_{1}$. Thus we have $\underline{u}_{\varepsilon}(t=0) \leq u_{\varepsilon}^{0}$ and

$$
\partial_{t} \underline{u}_{\varepsilon}-\left|\nabla \underline{u}_{\varepsilon}\right|^{2}-R\left(x, I_{\varepsilon}\right)-\varepsilon \Delta \underline{u}_{\varepsilon} \leq-\varepsilon C_{1}-4 \underline{L}_{1}^{2}|x|^{2}+\underline{K}_{2}|x|^{2}+\varepsilon 2 d \underline{L}_{1} \leq 0 .
$$

Consequently, we obtain that $u_{\varepsilon}(t, x) \geq-\underline{L}_{0}-\underline{L}_{1}|x|^{2}-\varepsilon\left(2 d \underline{L}_{1}\right) t$ for all $t \geq 0$ and $x \in \mathbb{R}^{d}$. Hence the estimates on $u_{\varepsilon}$. 
The next point is to show that the semi-convexity and the concavity of the initial data is preserved by equation (1). In other words, we are going to show the following lemma

Lemma 6.4. Under assumptions (44)-(50), we have for $t \geq 0$ and $x \in \mathbb{R}^{d}$

$$
-2 \underline{L}_{1} \leq D^{2} u_{\varepsilon}(t, x) \leq-2 \bar{L}_{1} .
$$

Proof. For a unit vector $\xi$, we use the notation $u_{\xi}:=\nabla_{\xi} u_{\varepsilon}$ and $u_{\xi \xi}:=\nabla_{\xi \xi}^{2} u_{\varepsilon}$ to obtain

$$
\begin{aligned}
& u_{\xi t}=R_{\xi}(x, I)+2 \nabla u \cdot \nabla u_{\xi}+\varepsilon \Delta u_{\xi}, \\
& u_{\xi \xi t}=R_{\xi \xi}(x, I)+2 \nabla u_{\xi} \cdot \nabla u_{\xi}+2 \nabla u \cdot \nabla u_{\xi \xi}+\varepsilon \Delta u_{\xi \xi} .
\end{aligned}
$$

By using $\left|\nabla u_{\xi}\right| \geq\left|u_{\xi \xi}\right|$ and the definition $\underline{w}(t, x):=\min _{\xi} u_{\xi \xi}(t, x)$ we arrive at the inequality

$$
\partial_{t} \underline{w} \geq-2 \underline{K}_{2}+2 \underline{w}^{2}+2 \nabla u \cdot \nabla \underline{w}+\varepsilon \Delta \underline{w} .
$$

And finally by a comparison principle and assumptions (49) and (50), we obtain

$$
\underline{w} \geq-2 \underline{L}_{1} .
$$

Hence the uniform semi-convexity of $u_{\varepsilon}$.

To prove the uniform concavity, we first recall that, at every point $(t, x) \in \mathbb{R}^{+} \times \mathbb{R}^{d}$, we can choose an orthonormal basis such that $D^{2} u_{\varepsilon}(t, x)$ is diagonal. Thus we can estimate the mixed second derivatives in terms of $u_{\xi \xi}$ and consequently we have

$$
\left|\nabla u_{\xi}\right|=\left|u_{\xi \xi}\right|
$$

By defining $\bar{w}(t, x):=\max _{\xi} u_{\xi \xi}(t, x)$ and using assumptions (46) and (62), we obtain the following inequality

$$
\partial_{t} \bar{w} \leq-2 \bar{K}_{2}+2 \bar{w}^{2}+2 \nabla u \cdot \nabla \bar{w}+\varepsilon \Delta \bar{w} .
$$

By a comparison principle and assumption we obtain the estimate

$$
\bar{w} \leq-2 \bar{L}_{1}
$$

which ends the proof of Lemma 6.4.

\section{2 $B V$ estimates on $\rho_{\varepsilon}^{2}, S_{\varepsilon}$ and their limits}

We use exactly the same proof as in Section 3 to obtain $B V$ estimates on $\rho_{\varepsilon}^{2}$ and $S_{\varepsilon}$. To obtain these estimates, an important point was the bounds on $\varepsilon P_{\varepsilon}$. We need to confirm that $\varepsilon P_{\varepsilon}$ is bounded, which was clear in Section 3 thanks to the bounds on the growth rate. Here the growth rate has a quadratic decrease at infinity, which does not give an 
immediate lower bound on $\varepsilon P_{\varepsilon}$. Furthermore we do not have a lower bound on $\rho_{\varepsilon}$ either because of the same argument and we cannot obtain directly a $B V$ estimate on $S_{\varepsilon}$ as in Section 3.2. However we derive a lower bound for $\varepsilon P_{\varepsilon}$ and we use the uniform concavity of $u_{\varepsilon}$ for that purpose.

By definition of $P_{\varepsilon}$, it follows from (44) and (59) that

$$
\begin{aligned}
\varepsilon P_{\varepsilon}=\int_{\mathbb{R}^{d}} n_{\varepsilon} R\left(x, S_{\varepsilon}(t)\right) d x & \geq \int_{\mathbb{R}^{d}} e^{\frac{1}{\varepsilon}\left(-\underline{L}_{0}-\underline{L}_{1}|x|^{2}-\varepsilon C_{1} t\right)}\left(-\underline{K}_{2}|x|^{2}\right) d x, \\
& \geq-\underline{K}_{2} e^{\frac{1}{\varepsilon}\left(-\underline{L}_{0}-\varepsilon C_{1} t\right)} \int_{\mathbb{R}^{d}} e^{-\frac{1}{\varepsilon} \underline{L}_{1}|x|^{2}}|x|^{2} d x, \\
& =-\underline{K}_{2} e^{\frac{1}{\varepsilon}\left(-\underline{L}_{0}-\varepsilon C_{1} t\right)} \frac{d \varepsilon}{2 \underline{L}_{1}}\left(\sqrt{\frac{\pi \varepsilon}{\underline{L}_{1}}}\right)^{d-1} .
\end{aligned}
$$

And we have a bound for $\left(\varepsilon P_{\varepsilon}\right)_{-}$.

We recall inequality (17) that also holds true in this framework

$$
\varepsilon \frac{d}{d t}\left(P_{\varepsilon}\right)_{-}+\alpha_{\varepsilon} \frac{\left(P_{\varepsilon}\right)_{-}}{\beta \mu_{\varepsilon}} \leq \bar{K}_{1} \rho_{M}\left(P_{\varepsilon}(0)+\beta \mu_{\varepsilon}(0) J_{\varepsilon}(0)\right)_{-} \frac{e^{\frac{-\mu_{m} K_{Q}}{\varepsilon}} t}{\beta \mu_{m}}+C \varepsilon .
$$

Then, we integrate this inequality over $[0, T]$ for $T>0$ and by the same arguments used in Section 3.1 it follows that $\rho_{\varepsilon}^{2}$ has local $B V$ bounds and therefore there exists a function $\bar{\rho}$ such that after extraction of a subsequence

$$
\rho_{\varepsilon} \longrightarrow \bar{\rho} \quad \text { in } L_{l o c}^{1}(0, \infty) \text {. }
$$

The next aim is to show that $S_{\varepsilon}$ has local BV bounds. We go back to equation (14) and we recall

$$
\varepsilon \beta \frac{d}{d t} J_{\varepsilon}=Q_{S} J_{e}+Q_{\rho} P_{\varepsilon}
$$

Then we have the following inequality

$$
\varepsilon \beta \frac{d}{d t}\left(-J_{\varepsilon}\right) \leq Q_{S}\left(-J_{\varepsilon}\right)+L_{Q}\left|P_{\varepsilon}\right|
$$

and

$$
\varepsilon \beta \frac{d}{d t}\left(J_{\varepsilon}\right)_{-} \leq Q_{S}\left(J_{\varepsilon}\right)_{-}+L_{Q}\left(\left(P_{\varepsilon}\right)_{+}+\left(P_{\varepsilon}\right)_{-}\right) .
$$

By integrating this inequality over $[0, T]$ for $T>0$, using

$$
\int_{0}^{T} L_{Q}\left|P_{\varepsilon}\right| \leq L_{Q}\left(\int_{0}^{T}\left(\dot{\rho}_{\varepsilon}\right)_{+}+\int_{0}^{T}\left(P_{\varepsilon}\right)_{-}\right)
$$


and since $\rho_{\varepsilon}$ is bounded above, we deduce from (17) that

$$
\int_{0}^{T}\left(J_{\varepsilon}\right)_{-} \leq C_{1} T+\underset{\varepsilon \rightarrow 0}{o}(1) .
$$

To conclude, we can extract a subsequence from $S_{\varepsilon}$ which locally converges in $L_{l o c}^{1}(0, \infty)$ to a limit function $\bar{S}$.

\subsection{The limit of the Hamilton-Jacobi equation}

From the estimates obtained above on $u_{\varepsilon}$ and $D^{2} u_{\varepsilon}$, we can deduce that $\nabla u_{\varepsilon}$ is locally uniformly bounded and thus from (27) for $\varepsilon<\varepsilon_{0}$ that $\partial_{t} u_{\varepsilon}$ is also locally uniformly bounded. Therefore there exists a function $u$ such that, after extraction of a subsequence (see [10, 26] for compactness properties), we have for $T>0$

$$
\begin{gathered}
u_{\varepsilon}(t, x) \underset{\varepsilon \rightarrow 0}{\longrightarrow} u(t, x) \text { strongly in } L^{\infty}\left(0, T ; W_{l o c}^{1, \infty}\left(\mathbb{R}^{d}\right)\right), \\
u_{\varepsilon}(t, x) \underset{\varepsilon \rightarrow 0}{\longrightarrow} u(t, x) \text { weakly-* in } L^{\infty}\left(0, T ; W_{l o c}^{2, \infty}\left(\mathbb{R}^{d}\right)\right) \cap W^{1, \infty}\left(0, T ; L_{l o c}^{\infty}\left(\mathbb{R}^{d}\right)\right),
\end{gathered}
$$

and

$$
\begin{gathered}
-\underline{L}_{0}-\underline{L}_{1}|x|^{2} \leq u(t, x) \leq \bar{L}_{0}-\bar{L}_{1}|x|^{2}+\bar{K}_{0} t, \quad-2 \underline{L}_{1} \leq D^{2} u(t, x) \leq-2 \bar{L}_{1} \quad \text { a.e. } \\
u \in W_{\mathrm{loc}}^{1, \infty}\left(\mathbb{R}^{+} \times \mathbb{R}^{d}\right) .
\end{gathered}
$$

Then, passing to the limit as $\varepsilon \rightarrow 0$ in equation (27), we deduce that $u$ satisfies in the viscosity sense the equation

$$
\left\{\begin{array}{l}
\frac{\partial}{\partial t} u=R(x, \bar{S}(t))+|\nabla u|^{2} \\
\max _{\mathbb{R}^{d}} u(t, x)=0
\end{array}\right.
$$

In particular $u$ is strictly concave, therefore it has exactly one maximum. This proves $n$ stays monomorphic and characterizes the Dirac location by

$$
\max _{\mathbb{R}^{d}} u(t, x)=0=u(t, \bar{x}(t)) .
$$

This completes the proof of Theorem 6.1. 


\subsection{The canonical equation}

In this section, we establish from the regularity properties proved in the previous sections a form of the so-called canonical equation in the language of adaptive dynamics (see [15, 22]):

$$
\dot{\bar{x}}(t)=\left(-D^{2} u(t, \bar{x}(t))\right)^{-1} \cdot \nabla_{x} R(\bar{x}(t), \bar{S}(t)) .
$$

This equation was formally introduced in [24] and holds true in our framework. The point of this differential equation is to describe the long time behaviour of the concentration point $\bar{x}(t)$.

First step: Bounds on third derivatives of $u_{\varepsilon}$. For the unit vectors $\xi$ and $\eta$, we use the notation $u_{\xi}:=\nabla_{\xi} u_{\varepsilon}, u_{\xi \eta}:=\nabla_{\xi \eta}^{2} u_{\varepsilon}$ and $u_{\xi \xi \eta}:=\nabla_{\xi \xi \eta}^{3} u_{\varepsilon}$ to derive

$$
\partial_{t} u_{\xi \xi \eta}=4 \nabla u_{\xi \eta} \cdot \nabla u_{\xi}+2 \nabla u_{\eta} \cdot \nabla u_{\xi \xi}+2 \nabla u \cdot \nabla u_{\xi \xi \eta}+R_{\xi \xi \eta}+\varepsilon \Delta u_{\xi \xi \eta} .
$$

Let us define

$$
M_{1}(t):=\max _{x, \xi, \eta} u_{\xi \xi \eta}(t, x) .
$$

Again, at every $(t, x) \in \mathbb{R}_{+} \times \mathbb{R}^{d}$, we can choose an orthogonal basis such that $D^{2}\left(\nabla_{\eta} u_{\varepsilon}(t, x)\right)$ is diagonal. And since $-u_{\xi \xi \eta}(t, x)=\nabla_{-\eta} u_{\xi \xi}(t, x)$, we have $M_{1}(t)=\max _{x, \xi, \eta}\left|u_{\xi \xi \eta}(t, x)\right|$. Then we obtain the following inequality

$$
\frac{d}{d t} M_{1} \leq 4 d M_{1}\left\|D^{2} u_{\varepsilon}\right\|_{\infty}+2 d M_{1}\left\|D^{2} u_{\varepsilon}\right\|_{\infty}+R_{\xi \xi \eta} .
$$

As assumption $(52)$ gives a bound on $M_{1}(t=0)$, by using the Grönwall lemma we obtain a $L^{\infty}$-bound on the third derivative uniform in $\varepsilon$.

Second step : Maximum point of $u_{\varepsilon}$ We denote the maximum point of $u_{\varepsilon}(t, \cdot)$ by $\bar{x}_{\varepsilon}(t)$. Since we have $\nabla u_{\varepsilon}\left(t, \bar{x}_{\varepsilon}(t)\right)=0$, we obtain

$$
\frac{d}{d t} \nabla u_{\varepsilon}\left(t, \bar{x}_{\varepsilon}(t)\right)=0
$$

Then the chain rule gives

$$
\frac{\partial}{\partial t} \nabla u_{\varepsilon}\left(t, \bar{x}_{\varepsilon}(t)\right)+D_{x}^{2} u_{\varepsilon}\left(t, \bar{x}_{\varepsilon}(t)\right) \dot{\bar{x}}_{\varepsilon}(t)=0
$$

and using equation (58), it follows that, for all $t \geq 0$, we have

$$
D_{x}^{2} u_{\varepsilon}\left(t, \bar{x}_{\varepsilon}(t)\right) \dot{\bar{x}}_{\varepsilon}(t)=-\frac{\partial}{\partial t} \nabla u_{\varepsilon}\left(t, \bar{x}_{\varepsilon}(t)\right)=-\nabla_{x} R\left(\bar{x}_{\varepsilon}(t), S_{\varepsilon}(t)\right)-\varepsilon \Delta \nabla_{x} u_{\varepsilon} .
$$

Thanks to the uniform bound on $D^{3} u_{\varepsilon}$ and the regularity on $R$, we pass to the limit

$$
\dot{\bar{x}}(t)=\left(-D^{2} u(t, \bar{x}(t))\right)^{-1} \cdot \nabla_{x} R(\bar{x}(t), \bar{S}(t)) \quad \text { a.e. }
$$


As we have $R(\bar{x}(t), \bar{S}(t))=0$ and assumption (44), $\bar{x}(t)$ is bounded in $L^{\infty}\left(\mathbb{R}_{+}\right)$. Then it implies from the canonical equation that $\bar{x}(t)$ is bounded in $W^{1, \infty}\left(\mathbb{R}_{+}\right)$and $\bar{S}(t)$ is also bounded in $W^{1, \infty}\left(\mathbb{R}^{d}\right)$ since $S \mapsto R(\cdot, S)$ is invertible by the Implicit Function Theorem. We differentiate (55) and obtain the following differential equation

$$
\dot{\bar{x}}(t) \cdot \nabla_{x} R+\dot{\bar{S}}(t) \nabla_{S} R=0
$$

Third step: Long time behaviour. Using the canonical equation we obtain

$$
\begin{aligned}
\frac{d}{d t} R(\bar{x}(t), \bar{S}(t)) & =\nabla R(\bar{x}(t), \bar{S}(t)) \frac{d}{d t} \bar{x}(t)+\partial_{S} R(\bar{x}(t), \bar{S}(t)) \frac{d}{d t} \bar{S}(t) \\
& =\nabla R(\bar{x}(t), \bar{S}(t))\left(-D^{2} u\right)^{-1} \nabla R(\bar{x}(t), \bar{S}(t))+\partial_{S} R(\bar{x}(t), \bar{S}(t)) \frac{d}{d t} \bar{S}(t) .
\end{aligned}
$$

Since the left hand side equals 0 from (55), it follows that

$$
\frac{d}{d t} \bar{S}(t)=\frac{-1}{\partial_{S} R(\bar{x}(t), \bar{S}(t))} \nabla R(\bar{x}(t), \bar{S}(t))\left(-D^{2} u\right)^{-1} \nabla R(\bar{x}(t), \bar{S}(t)) \leq 0 .
$$

We deduce that $\bar{S}(t)$ decreases. Consequently $\bar{S}(t)$ converges and subsequences of $\bar{x}(t)$ also converge since $\bar{x}(t)$ is bounded. However the possible limits $\bar{x}_{\infty}$ and $\bar{S}_{\infty}$ have to satisfy $\nabla R\left(\bar{x}_{\infty}, \bar{S}_{\infty}\right)=0$. Then from (43), 45) and (55), we conclude that

$$
\bar{S}(t) \underset{t \rightarrow \infty}{\longrightarrow} S_{m}, \quad \bar{x}(t) \underset{t \rightarrow \infty}{\longrightarrow} \bar{x}_{\infty}=0,
$$

which ends the proof of Theorem 6.2 .

\section{Numerical results and discussion}

We illustrate in this section the evolution of $n_{\varepsilon}, \rho_{\varepsilon}$ and $S_{\varepsilon}$ in time with different values of $\beta$. We choose the following initial data

$$
n^{0}=C_{\text {mass }} \exp \left(-(x-0.8)^{2} / \varepsilon\right),
$$

and growth rate $R$ and $Q$ as follows

$$
\begin{gathered}
R(x, S)=0.2\left(-0.6+0.3 S-(x-0.5)^{2}\right), \\
Q(\rho, S)=10-(1.5+\rho) S .
\end{gathered}
$$

The numerics have been performed in Matlab with parameters as follows. We consider the solution on interval $[0,1]$. We use a uniform grid with 1000 points on the segment and denote by $n_{i}^{k}$ and $S^{k}$ the numerical solutions at grid point $x_{i}=i \Delta x$ and at time $t_{k}=k \Delta t$. We choose as initial value of the nutrient concentration $S_{\varepsilon}(t=0)=5$. We also choose $\beta$ to be $2.10^{3}$, the time step $\Delta t=10^{-4}$ and $C_{\text {mass }}$ such as the initial mass of the population 

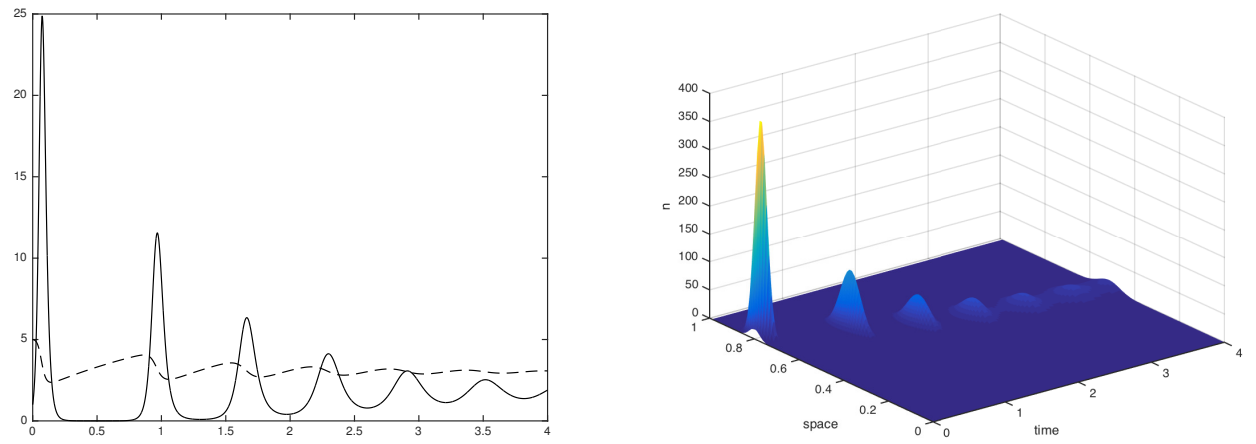

Figure 1: Dynamics of $\rho_{\varepsilon}$ (_ $)$ and $S_{\varepsilon}\left({ }--_{-}\right)$(left) and dynamics of the density $n_{\varepsilon}$ for $\beta=2 \cdot 10^{3}$ and $\varepsilon=10^{-3}$.
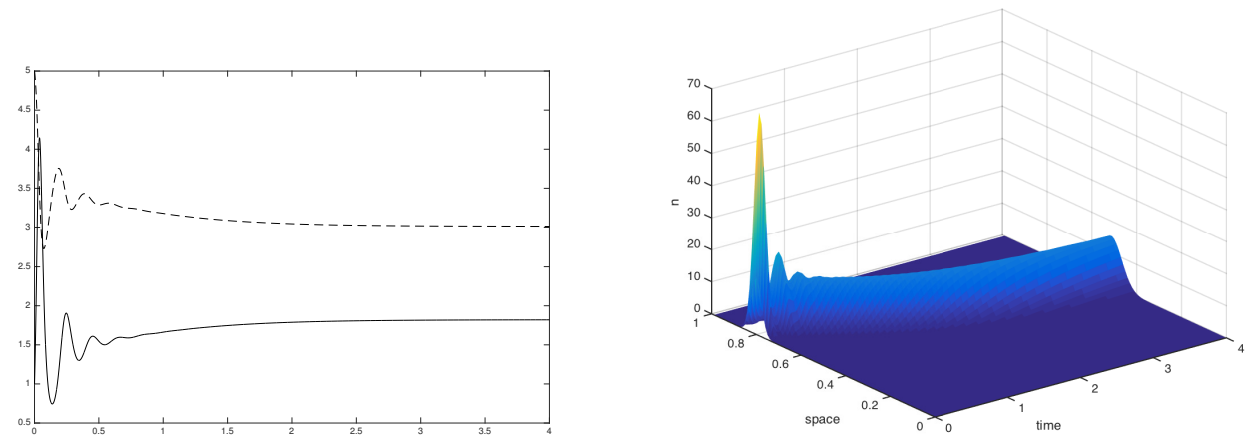

Figure 2: Dynamics of $\rho_{\varepsilon}$ (_ $)$ and $S_{\varepsilon}(----)$ (left) and dynamics of the density $n_{\varepsilon}$ for $\beta=2 \cdot 10^{2}$ and $\varepsilon=10^{-3}$

in the computational domain is equal to 1 . The equation is solved by an implicit-explicit finite-difference method.

The Figure 1 shows the dynamics for $\varepsilon=1 \cdot 10^{-3}$ and the Figure 2 for $\varepsilon=5 \cdot 10^{-4}$. In Figure 3, we show the numerical results corresponding to the same data as in Figure 1, except that we choose $\beta=2 \cdot 10^{2}$. We can observe oscillations of $\rho_{\varepsilon}$ and $S_{\varepsilon}$ in the first case $\left(\beta=2 \cdot 10^{3}\right)$, whereas there are very few variations of these quantities when $\beta$ is smaller.

Some open questions arise from the present study. First it seems that the method developed in this work does not give TV bounds for the full range $\left[0, \beta_{0}\right]$ for some small $\beta_{0}$ since the estimations providing the uniform $B V$ estimates on $\rho^{2}$ in Section 3.2 are local and then it is not possible to prove uniform convergence of $S(t)$ as $\beta \rightarrow 0$ on $[0, \infty)$ at this stage. Thus we cannot obtain the asymptotic behaviour of the limit functions as $\beta$ goes to 0 , while the convergence of $\varepsilon$ to 0 describes the dynamics of the presented system in a 
larger time scale, therefore local estimates are enough.

As mentioned in Section 4, the uniqueness of the solution to the Hamilton-Jacobi equation (71) has up to now been an open problem, apart from very particular cases (see for instance [6]). However a recent work of S. Mirrahimi and J. Roquejoffre [40] has shown uniqueness of the constrained Hamilton-Jacobi equation related to the following selectionmutation model in the concavity framework

$$
\begin{gathered}
\varepsilon \partial_{t} n_{\varepsilon}(t, x)=n_{\varepsilon}(t, x) R\left(x, I_{\varepsilon}(t)\right)+\varepsilon^{2} \Delta n_{\varepsilon}(t, x), \\
I_{\varepsilon}(t)=\int_{\mathbb{R}^{d}} \psi(x) n_{\varepsilon}(t, x) d x,
\end{gathered}
$$

which could be a first step to prove uniqueness for the presented chemostat model.

\section{Acknowledgements}

The authors have been supported by ANR-13-BS01-0004 funded by the French Ministry of Research (ANR Kibord).

\section{References}

[1] Azmy S. Ackleh, Ben G. Fitzpatrick, and Horst R. Thieme. Rate distributions and survival of the fittest: a formulation on the space of measures. Discrete Contin. Dyn. Syst. Ser. B, 5(4):917-928 (electronic), 2005.

[2] M. Bardi and I. Capuzzo-Dolcetta. Optimal control and viscosity solutions of Hamilton-Jacobi-Bellman equations. Birkhäuser Boston, 1997.

[3] G. Barles. Solutions de viscosité des équations de Hamilton-Jacobi. Springer-Verlag Berlin Heidelberg, 1994.

[4] G. Barles, L. C. Evans, and P. E. Souganidis. Wavefront propagation for reaction diffusion systems of pde. Duke Math. J., 61(3):835-858, pages 835-858, 1990.

[5] G. Barles, S. Mirrahimi, and B. Perthame. Concentration in Lotka-Volterra parabolic or integral equations: a general convergence result. Methods Appl. Anal., 16(3):321$340,2009$.

[6] G. Barles and B. Perthame. Concentrations and constrained hamilton-jacobi equations arising in adpative dynamics. Contemporary Mathematics, 439:57, 2007.

[7] G. Barles and B. Perthame. Dirac concentrations in Lotka-Volterra parabolic PDEs. Indiana Univ. Math J., 57 (7):3275-3301, 2008. 
[8] F. Bethuel, H. Brézis, and F. Helein. Ginzburg-Landau vortices. Progress in Nonlinear Differential Equations and Their Applications. Birkhäuser Boston, 1994.

[9] E. Bouin and S. Mirrahimi. A hamilton-jacobi limit for a model of population stuctured by space and trait. Comm. Math. Sci., 13.6:1431-1452, 2015.

[10] H. Brézis. Functional Analysis, Sobolev Spaces and Partial Differential Equations. Springer Science, 2010.

[11] H. Brézis and A. Friedman. Nonlinear parabolic equations involving measures as initial conditions. J. Math. Pures App. (9), 62(1):73-97, 1983.

[12] Nicolas Brunel and Vincent Hakim. Fast global oscillations in networks of integrateand-fire neurons with low firing rates. Neural computation, 11(7):1621-1671, 1999.

[13] J. A. Carrillo, S. Cuadrado, and B. Perthame. Adaptive dynamics via hamilton-jacobi approach and entropy methods for a juvenile-adult model. Mathematical Biosciences, 205(1):137-161, 2007.

[14] N. Champagnat. Mathematical study of stochastic models of evolution belonging to the ecological theory of adaptive dynamics. PhD thesis, Université de Nanterre-Paris $\mathrm{X}, 2004$.

[15] N. Champagnat, R. Ferrière, and G. Ben Arous. The canonical equation of adaptive dynamics: A mathematical view. Selection, 2(1):73-83, 2002.

[16] N. Champagnat, R. Ferrière, and S. Méléard. Unifying evolutionary dynamics: From individual stochastic processes to macroscopic models. Theoretical Population Biology, 69(3):297-321, 2006.

[17] N. Champagnat, R. Ferrière, and S. Méléard. Individual-based probabilistic models of adaptive evolution and various scaling approximations, volume 59 of Progress in Probability. Birkhaüser, 2008.

[18] N. Champagnat and P.-E. Jabin. The evolutionary limit for models of populations interacting competitively via several resources. Journal of Differential Equations, 251(1):176-195, 2011.

[19] N. Champagnat, P.-E. Jabin, and G. Raoul. Convergence to equilibrium in competitive Lotka-Volterra equations and chemostat systems. C. R. Acad. Sci. Paris Sér. I Math., 348(23-24), 1267-1272, 2010.

[20] M. G. Crandall, H. Ishii, and P.-L. Lions. User's guide to viscosity solutions of second order partial differential equations. Bulletin of the American Mathematical Society, 27(1):1-67, 1992. 
[21] L. Desvillettes, P.-E. Jabin, S. Mischler, and G.Raoul. On mutation selection dynamics. Commun. Math. Sci., 6(3):729-747, 2008.

[22] U. Dieckmann and R. Law. The dynamical theory of coevolution: a derivation from stochastic ecological processes. Journal of Mathematical Biology, 34(5-6):579-612, 1996.

[23] O. Diekmann. A beginner's guide to adaptive dynamics. Banach Center publications, 63:47-86, 2004.

[24] O. Diekmann, P.-E. Jabin, S. Mischler, and B. Perthame. The dynamics of adaptation: an illuminating example and a hamilton-jacobi approach. Theoretical Population Biology, 67(4):257-271, 2005.

[25] L. C. Evans and P. E. Souganidis. A pde approach to geometric optics for certain semilinear parabolic equations. Indiana Univ. Math J., 38(1):141-172, 1989.

[26] L.C. Evans. Partial differential equations, volume 19 of Graduate Studies in Mathematics. American Mathematical Society, Providence, RI, 1998.

[27] W. H. Fleming and H. M. Soner. Controlled Markov Processes and Viscosity Solutions. Applications of Mathematics 25. Springer, 1993.

[28] S. A. H. Geritz, E. Kisdi, G. Meszena, and J. A. J. Metz. Evolutionarily singular strategies and the adaptive growth and branching of the evolutionary tree. Evolutionary Ecology, 12(1):35-57, 1998.

[29] J. Hofbauer and K. Sigmund. Evolutionary games and population dynamics. Cambridge University Press, 1998.

[30] J. Hofbauer and K. Sigmund. Evolutionary game dynamics. Bulletin of the American Mathematical Society, 40(4):479-519, 2003.

[31] Freidlin M. I. Functional Integration and Partial Differential Equations. Number 109. Princeton University Press, 1985.

[32] Freidlin M. I. Limit theorems for large deviations and reaction-diffusion equations. The Annals of Probability, pages 639-675, 1985.

[33] A. Lorz, S. Mirrahimi, and B. Perthame. Dirac mass dynamics in multidimensional nonlocal parabolic equations. Communications in Partial Diffenretial Equations, 36(6):1071-1098, 2011.

[34] A. Lorz and B. Perthame. Long-term behaviour of phenotypically structured models. Proceedings of the Royal Society A: Mathematical, Physical and Engineering Science, 470(2167):20140089, 10, 2014. 
[35] G. Meszena, M. Gyllenberg, F. J. Jacobs, and J. A. J. Metz. Link between population dynamics and dynamics of darwinian evolution. Physical Review Letters, 95(7):078105, 2005.

[36] S. Mirrahimi. Phénomènes de concentration dans certaines EDPs issues de la biologie. $\mathrm{PhD}$ thesis, Université Pierre et Marie Curie-Paris VI, 2011.

[37] S. Mirrahimi. Adaptation and migration of a population between patches. Discrete and Continuous Dynamical System - B (DCDS-B), 18.3:753-768, 2013.

[38] S. Mirrahimi and B. Perthame. Asymptotic analysis of a selection model with space. To appear in J. Math. Pures Appl.

[39] S. Mirrahimi, B. Perthame, E. Bouin, and P. Millien. Population formulation of adaptative meso-evolution: theory and dynamics. In J. F. Rodrigues and F. Chalub, editors, The Mathematics of Darwin's Legacy, Mathematics and Biosciences in Interaction. Springer, 2011.

[40] S. Mirrahimi and J.-M. Roquejoffre. Uniqueness in a class of hamilton-jacobi equations with constraints. Comptes Rendus Mathématiques, 2015.

[41] B. Perthame. Transport equations in biology. Frontiers in Mathematics. Birkhäuser Verlag, Basel, 2007.

[42] G. Raoul. Etude qualitative et numérique d'équations aux dérivées partielles issues des sciences de la nature. PhD thesis, ENS Cachan, 2009.

[43] G. Raoul. Local stability of evolutionary attractors for continuous structured populations. Monatsh Math., 2010.

[44] H. L. Smith and P. Waltman. The theory of the chemostat: dynamics of microbial competition. Cambridge Univ. Press, 1994.

[45] J. Maynard Smith. Evolution and the Theory of Games. Cambridge Univ. Press, Cambridge, 1982. 